\title{
Carbonized cellulose beads for efficient capacitive energy storage
}

\author{
Chang-Qing Ruan $(\mathbb{D} \cdot$ Zhaohui Wang $\cdot$ Jonas Lindh $\cdot$ Maria Strømme
}

Received: 10 January 2018/Accepted: 25 April 2018/Published online: 28 April 2018

(C) The Author(s) 2018

\begin{abstract}
Natural biomaterials, including polysaccharides and amino acids, provide a sustainable source of functional carbon materials for electric energy storage applications. We present a one-pot reductive amination process to functionalize 2,3-dialdehyde cellulose (DAC) beads with chitosan and L-cysteine to provide single $(\mathrm{N})$ - and dual $(\mathrm{N} / \mathrm{S})$-doped materials. The functionalization enables the physicochemical properties of the materials to be tailored and can provide carbon precursors with heteroatom doping suitable for energy storage applications. Scanning electron microscopy, Fourier transform infrared spectroscopy and thermogravimetric analysis were used to characterize the changes to the beads after functionalization and carbonization. The results of X-ray photoelectron spectroscopy and energy-dispersive $\mathrm{X}$-ray spectroscopy verified that the doping was effective, while the nitrogen sorption isotherms and pore-size distributions of the carbonized beads showed the effects of doping with different hierarchical porosities. In the electrochemical experiments, three
\end{abstract}

C.-Q. Ruan · J. Lindh · M. Strømme

Nanotechnology and Functional Materials, Department of Engineering Sciences, The Ångström Laboratory, Uppsala University, Box 534, 75121 Uppsala, Sweden

Z. Wang $(\bowtie)$

Department of Chemistry-Ångström, The Ångström

Laboratory, Uppsala University, Box 538,

75121 Uppsala, Sweden

e-mail: Zhaohui.Wang@kemi.uu.se kinds of carbon beads [pyrolyzed from DAC, chitosan-crosslinked DAC (CS-DAC) and L-cysteinefunctionalized DAC] were used as electrode materials. Electrodes of carbonized CS-DAC beads had a specific capacitance of up to $242 \mathrm{~F} \mathrm{~g}^{-1}$ at a current density of $1 \mathrm{~A} \mathrm{~g}^{-1}$. These electrodes maintained a capacitance retention of $91.5 \%$ after 1000 charge/ discharge cycles, suggesting excellent cycling stability. The results indicate that reductive amination of DAC is an effective route for heteroatom doping of carbon materials to be used as electrode active materials for energy storage.

Keywords 2,3-Dialdehyde cellulose (DAC) beads . Nitrogen doping $\cdot$ Nitrogen/sulfur doping ·

Supercapacitor

\section{Introduction}

In the development of portable electronics such as digital telecommunication systems and systems using pulse laser techniques, the lack of lightweight, inexpensive, sustainable energy storage devices has been a limiting factor (Deng et al. 2016; Zhao et al. 2014b). Among the currently employed energy storage devices, supercapacitors, which are promising candidates because of their excellent cycling stabilities, higher power density than batteries, and higher energy 
density than conventional physical capacitors, have attracted significant research interest (Simon et al. 2014; Winter and Brodd 2004; Zong et al. 2016; Li et al. 2014, 2017a, b; You et al. 2017). Supercapacitors can be divided into two categories according to their charge storage mechanisms: pseudocapacitors and electrical double layer capacitors (EDLCs) (Wang et al. 2012; Winter and Brodd 2004). Pseudocapacitors store electrical energy by fast, reversible faradaic reactions (redox reactions, electrosorption or intercalation) occurring on the electrode materials, typically of metal oxides (Jeong et al. 2016; Wang et al. 2013a) or conducting polymers (Wang et al. 2014, 2015; Zhao et al. 2017). EDLCs are associated with an electrodepotential-dependent accumulation of electrostatic charge at the interface between the electrolyte and the electrode, typically of carbon (Ghosh and Lee 2012). The use of carbon here is merited by the many desirable properties of this material, such as low processing costs, chemical inertness, thermo-stability, non-toxicity, wide availability, high electrical conductivity, and a long and stable cycle life (Jiang et al. 2013; Xu et al. 2016; Zhai et al. 2011). Of the various carbon materials with different morphologies and distinct physical and chemical properties (Fan and Shen 2015; Zhai et al. 2011), carbon microspheres have several advantages, such as their regular spheroidal shape, and their adjustable porosity and particle size (which can decrease the resistance of ion diffusion and thus improve the electrochemical performance) (Duffy et al. 2012; Liu et al. 2013). Moreover, the macroporosity created as the carbon microspheres aggregate can promote the generation of ion-buffer reservoirs, which could decrease the diffusion distance of electrolyte ions to the carbon surface (Jiang et al. 2013; Zhao et al. 2014a). Hence, carbon microspheres have become one of the most promising electrode materials in supercapacitor research and development (Liu et al. 2013; Ma et al. 2014; Zhu et al. 2015). As a useful, simple approach, heteroatom doping (e.g. with nitrogen, sulfur, boron and/or phosphorus) is often used to modify the intrinsic electronic structure (Lee et al. 2012), enhance the wettability of the electrode surface (Zhu et al. 2015) or introduce new pseudocapacitive interactions (Biel et al. 2009) in order to improve the electrochemical performance of carbon electrodes (Chen et al. 2014b; $\mathrm{Li}$ et al. 2007). There are two main methods employed in heteroatom doping; one is by direct pyrolysis of heteroatom-containing precursors (Xu et al. 2015; Zhu et al. 2015) and the other is by post-treating carbons with dopants (Chen et al. 2014b; You et al. 2013).

Cellulose, the most abundant renewable biopolymer in nature (Klemm et al. 2005), is a promising pyrolysis precursor for providing carbonous species. Chitosan is a pseudonatural polysaccharide composed of glucosamine and $\mathrm{N}$-acetyl glucosamine (Rinaudo 2006). As chitosan is derived from chitin, the second most abundant and renewable biopolymer after cellulose, it is also almost inexhaustible (Rinaudo 2006). Chitosan is also a commonly used source of carbon species self-doped with nitrogen. Cysteine, a natural amino acid containing both nitrogen and sulfur, is a good candidate for providing nitrogen and sulfur doping; it has been used to functionalize carbohydrates in the production of dual-doped carbon microspheres (Wohlgemuth et al. 2012). Recently, work on the reductive amination and preparation of 2,3dialdehyde cellulose (DAC) beads (Lindh et al. 2014), L-cysteine-functionalized DAC (LC-DAC) beads (Ruan et al. 2016) and chitosan-crosslinked DAC (CS-DAC) beads (Ruan et al. 2018) has been reported by our group for use in applications such as palladium adsorption and Congo red dye adsorption. All three of these micrometer-sized biopolymer beads are interesting candidates for a carbon source, with or without heteroatom doping.

The aim of this study was to broaden the application of these cellulose beads to include energy storage by using DAC, CS-DAC and LC-DAC beads as a carbon source to provide microscale porous carbon beads doped with heteroatoms ( $\mathrm{N}$ and/or $\mathrm{S}$ ), or un-doped beads, for use as an electrode material for manufacturing supercapacitors.

\section{Experimental section}

\section{Materials}

Nanocellulose from Cladophora green algae was provided by FMC Biopolymer (Batch G-3095-10; USA). Sodium metaperiodate $\left(\mathrm{NaIO}_{4}\right)$, chitosan (low molecular weight, 75-85\% deacetylated), L-cysteine, sodium cyanoborohydride $\left(\mathrm{NaBH}_{3} \mathrm{CN}\right)$ and other chemicals used were of analytical or reagent grade and were purchased from Sigma-Aldrich. Deionized water was used for all the experimental procedures. 
Preparation of 2,3-dialdehyde cellulose (DAC) beads

Porous DAC beads were prepared according to a previously described method (Lindh et al. 2014) with minor modifications. Briefly, Cladophora nanocellulose $(20 \mathrm{~g})$ was dispersed in $1.8 \mathrm{~L}$ of water and mixed with sodium metaperiodate $(134 \mathrm{~g}, 5 \mathrm{~mol}$ per mol of anhydroglucose units) dissolved in $0.2 \mathrm{~L}$ water. The proposed periodate oxidation needs to be performed in the absence of light, which was catered for by covering the reaction vessel in aluminum foil. The reaction mixture was gently stirred at room temperature in the dark for 10 days. The reaction was then quenched via the addition of ethylene glycol and the mixture was washed four times by centrifugation/washing/re-dispersion in water to provide pure DAC. Never-dried DAC samples were kept in water at $4{ }^{\circ} \mathrm{C}$ for further use.

Preparation of chitosan-functionalized DAC (CSDAC) beads

The CS-DAC beads were prepared according to a previously described procedure (Ruan et al. 2018). In short, never-dried DAC beads (about $500 \mathrm{mg}$ dried sample) were dispersed in distilled water $(250 \mathrm{~mL})$ to obtain a DAC bead suspension with good dispersibility at a concentration of about $2 \mathrm{mg} \mathrm{mL}^{-1}$. Chitosan $(960 \mathrm{mg})$ was dissolved in $\mathrm{HCl}$ solution $(50 \mathrm{~mL})$ to a concentration of $19.2 \mathrm{mg} \mathrm{mL}^{-1}$ and the $\mathrm{pH}$ of the solution was adjusted to be in the range of 2.5-3.5. The chitosan solution was added drop-wise (about $1 \mathrm{~mL} \min ^{-1}$ ) into the DAC bead suspension in a round-bottomed flask followed by the addition of the reducing agent $\mathrm{NaBH}_{3} \mathrm{CN}(1.25 \mathrm{~mol}$ per mol of chitosan). The Schiff base reaction and reduction reaction were carried out at room temperature. The reaction was stopped by centrifugation after $24 \mathrm{~h}$ and the products were washed twice by centrifugation/ washing/redispersion with $\mathrm{HCl}$ solution $(10 \mathrm{mM})$, twice by centrifugation/washing/redispersion with distilled water and twice by centrifugation/washing/ redispersion with ethanol. The purified sample was dried in a vacuum oven at $45{ }^{\circ} \mathrm{C}$ overnight.
Preparation of L-cysteine-functionalized DAC (LC-DAC) beads

The LC-DAC beads were prepared as previously published (Ruan et al. 2016). Briefly, never-dried DAC beads (amount estimated to be equivalent to about $500 \mathrm{mg}$ dried sample) were dispersed in acetate buffer solution $(250 \mathrm{~mL}, 0.1 \mathrm{M}, \mathrm{pH}$ about 4.5$)$ to obtain a DAC bead suspension with good dispersibility at a concentration of about $2 \mathrm{mg} \mathrm{mL}^{-1}$. L-cysteine (757 mg) was also dissolved in acetate buffer solution (50 mL, $0.1 \mathrm{M}, \mathrm{pH}$ about 4.5 ) to a concentration of about $15.1 \mathrm{mg} \mathrm{mL}^{-1}$. This solution was then added drop-wise into the DAC bead suspension at a controlled speed of about $1 \mathrm{~mL} \mathrm{~min}{ }^{-1}$ followed by the addition of the $\mathrm{NaBH}_{3} \mathrm{CN}$ reducing agent (1.25 mol per mol of chitosan). The Schiff base and reduction reactions were performed at room temperature. The reaction was stopped by centrifugation after $24 \mathrm{~h}$ and the products were washed four times by centrifugation/washing/redispersion with distilled water and twice by centrifugation/washing/redispersion with ethanol. The purified samples were dried in a vacuum oven at $45{ }^{\circ} \mathrm{C}$ overnight.

Preparation of carbon microspheres from DAC, CS-DAC and LC-DAC

All as-prepared beads were pyrolyzed under a nitrogen flow at $400{ }^{\circ} \mathrm{C}$ (at a heating rate of $5^{\circ} \mathrm{C} \mathrm{min}^{-1}$ ) for $1 \mathrm{~h}$ and then at $900{ }^{\circ} \mathrm{C}$ for $1 \mathrm{~h}$ (at a heating rate of $5{ }^{\circ} \mathrm{C} \mathrm{min}^{-1}$ ) and were then cooled to room temperature in a nitrogen atmosphere.

Scanning electron microscopy (SEM) and energy dispersive X-ray (EDX) analysis

The morphology of the produced materials was visualized in a LEO 1550 field-emission SEM instrument (Zeiss, Germany) at an accelerating voltage of $1 \mathrm{kV}$, with an in-lens secondary electron detector. EDX analysis was performed on the same LEO 1550 field-emission SEM instrument (Zeiss, Germany) but at an accelerating voltage of $20 \mathrm{kV}$, with a map model to obtain the relative content of the elements in the samples. 
Nitrogen sorption isotherm analysis

The specific surface area (SSA) of all samples was recorded with an ASAP 2020 instrument (Micromeritics, USA) using a multipoint Brunauer-EmmettTeller approach (Brunauer et al. 1938) involving $\mathrm{N}_{2}$ gas adsorption isotherm analysis. The pore size distribution was determined using density function theory (DFT) with DFT plus software from Micromeritics.

\section{Fourier transform infrared (FTIR) spectroscopy}

FTIR spectra were recorded on a Tensor 27 FTIR spectrometer (Bruker, Germany) with an attenuated total reflectance sample holder. The resolution was set to $4 \mathrm{~cm}^{-1}$ with 128 scans over a range of 2000-800 $\mathrm{cm}^{-1}$.

Thermal gravimetric analysis (TGA)

TGA was carried out on a Mettler Toledo instrument, model TGA/SDTA851, under nitrogen flow, in an inert aluminum oxide cup. The samples were heated from 30 to $900{ }^{\circ} \mathrm{C}$ at a heating rate of $5^{\circ} \mathrm{C} \mathrm{min}^{-1}$.

\section{X-ray photoelectron spectroscopy (XPS)}

High-resolution XPS spectra were prepared on an ESCA instrument (Physical Electronics, USA) equipped with an $\mathrm{Al} \mathrm{K} \alpha$ source $(1486.6 \mathrm{eV}, 45 \mathrm{~W})$; the analyzer was operated at $23.5 \mathrm{eV}$ pass energy.

Raman spectroscopy analysis

Raman spectroscopy analyses were carried out with a Renishaw Ramascope equipped with a Leica LM optical microscope and an argon ion laser (1 $1 / 4$ $514.5 \mathrm{~nm}$ ) source.

Electrochemical characterization and analysis

Carbon electrodes composed of carbonized cellulose beads with a polyvinylidene fluoride binder in a ratio of 9:1 by weight were prepared by casting the obtained slurry onto graphite foil. The electrodes were then dried at $80{ }^{\circ} \mathrm{C}$ overnight in a vacuum oven. The carbon electrodes had a typical active mass loading of $1 \mathrm{mg} \mathrm{cm}^{-2}$.
All electrochemical experiments were performed at room temperature using an Autolab/GPES instrument (ECO Chemie, The Netherlands). For cyclic voltammetry $(\mathrm{CV})$ measurements, the as-obtained carbon electrodes were used as the working electrode in a 3-electrode electrochemical cell; the other two electrodes were a $\mathrm{Pt}$ wire counter electrode and an $\mathrm{Ag}$ / $\mathrm{AgCl}$ reference electrode. An aqueous $1 \mathrm{M} \mathrm{H}_{2} \mathrm{SO}_{4}$ solution, which was purged with $\mathrm{N}_{2}$ for 10 min prior to the measurements, was used as the electrolyte.

Two-electrode symmetric supercapacitor devices hermetically heat-sealed in laminated aluminum foil were made as previously described (Wang et al. 2014, 2015). A piece of filter paper, pre-soaked with $1.0 \mathrm{M} \mathrm{H}_{2} \mathrm{SO}_{4}$ solution and used as the separator, was sandwiched between two carbon electrodes.

Galvanostatic charge-discharge measurements were carried out at different current densities between 0.5 and $20 \mathrm{~A} \mathrm{~g}^{-1}$ with a charging voltage of $0.8 \mathrm{~V}$. The specific gravimetric electrode capacitance $\left(\mathrm{C}_{\mathrm{g}}\right)$ was derived from the galvanostatic discharge curves using the formula: $C_{g}=4 I \Delta t /(m \Delta V)$, where $I$ denotes the discharge current, $\Delta V$ represents the potential change during the discharge time $\Delta t$, and $m$ represents the total mass of carbon in the two electrodes.

\section{Results and discussion}

Preparation and characterization of CS-DAC and LC-DAC beads

DAC, CS-DAC and LC-DAC beads were prepared according to previously published work (Lindh et al. 2014; Ruan et al. 2016, 2018), with minor modifications. As shown in Scheme 1, the alcohol groups at C2 and $\mathrm{C} 3$ of Cladophora cellulose were oxidized by $\mathrm{NaIO}_{4}$, resulting in the formation of dialdehyde groups. Porous spherical DAC beads with a diameter of a few micrometers were obtained after washing with ethanol (Fig. 1a). A Schiff base reaction took place between the aldehyde groups in the DAC and primary amine groups in chitosan and L-cysteine to provide imine $(-\mathrm{C}=\mathrm{N}-$ ) groups (not shown in Scheme 1), which were reduced by $\mathrm{NaBH}_{3} \mathrm{CN}$ in situ to generate an amine. The porous morphology of CS-DAC and LC-DAC beads remained, as shown in the SEM images (Fig. 1b, c), after the reductive amination; this is similar to the surface morphology 


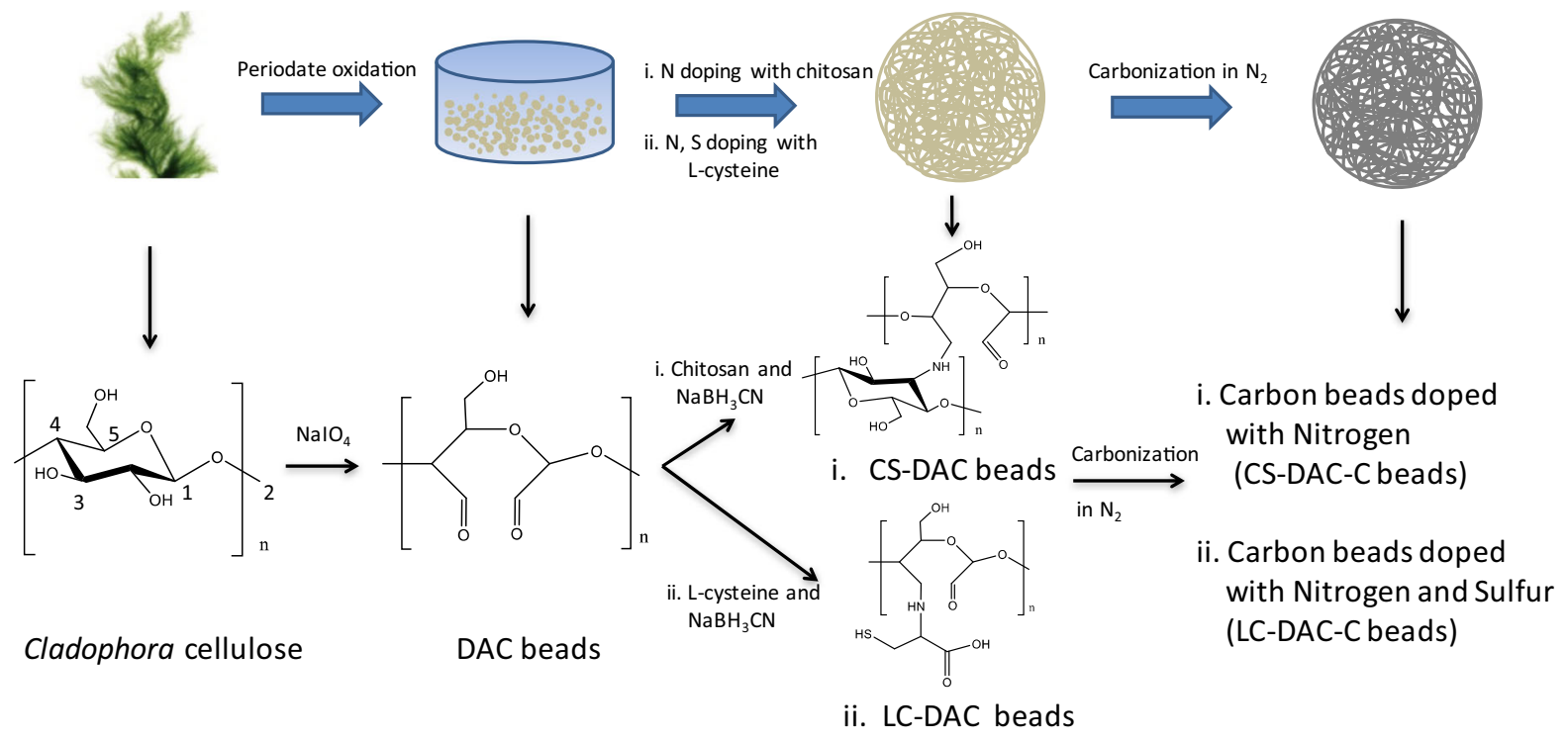

Scheme 1 Preparation process for doping carbonized chitosan-crosslinked 2,3-dialdehyde cellulose (CS-DAC-C) and L-cysteinefunctionalized DAC (LC-DAC-C) beads
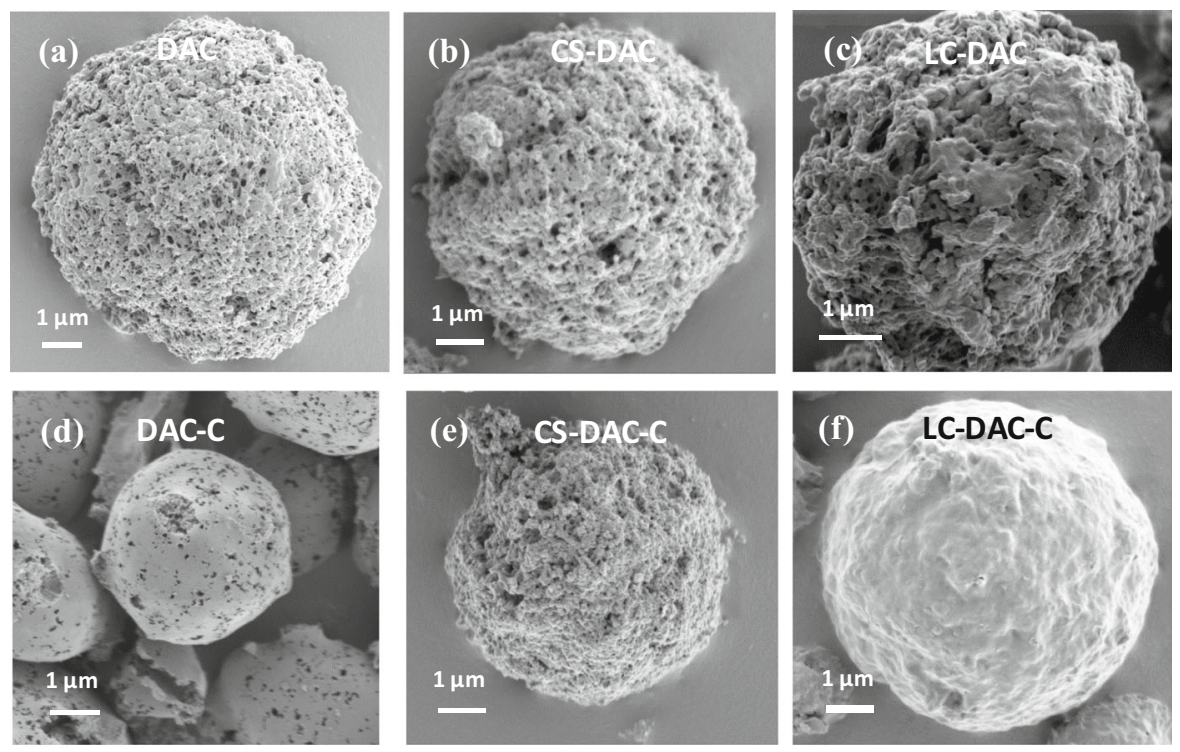

Fig. 1 Scanning electron microscopy images of a 2,3-dialdehyde cellulose (DAC), b chitosan-crosslinked DAC (CS-DAC), c L-cysteine-functionalized DAC (LC-DAC), d carbonized

previously reported (Ruan et al. 2016, 2018). No obvious change was found after the functionalization with chitosan, but the beads modified with cysteine looked slightly more compact on the surface.

FTIR spectra (Fig. 2a) revealed some differences between cellulose, DAC, CS-DAC and LC-DAC, such as the absorbance peak at $1730 \mathrm{~cm}^{-1}$ assigned to
DAC (DAC-C), e carbonized CS-DAC (CS-DAC-C), and f carbonized LC-DAC (LC-DAC-C) beads

carbonyl groups, which appeared in DAC because aldehyde groups were produced after the oxidation of cellulose. However, the Schiff base and reduction reactions between DAC and chitosan consumed the aldehyde groups so that this absorbance peak disappeared in CS-DAC. Although similar reactions took place between DAC and L-cysteine, a strong 

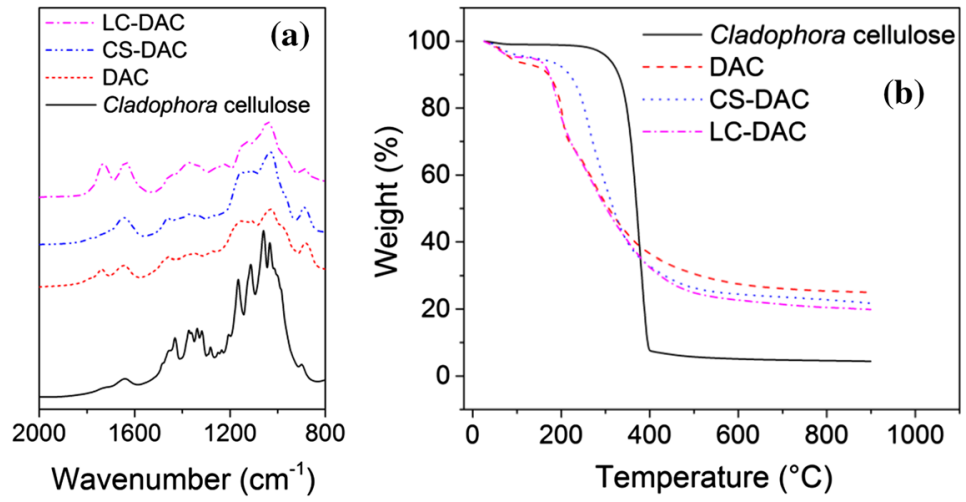

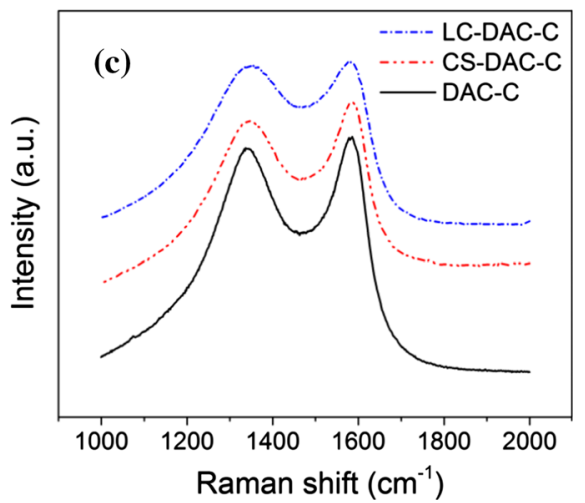

Fig. 2 a Fourier-transform infrared spectroscopy spectra and b thermogravimetric analysis curves of non-carbonized 2,3dialdehyde cellulose (DAC), chitosan-crosslinked DAC (CS-

absorbance peak at $1730 \mathrm{~cm}^{-1}$ was still observed in the LC-DAC beads; this was the result of the newly introduced carboxylic groups in L-cysteine.

The TGA curves (Fig. 2b) showed that DAC beads degraded at significantly lower temperatures than cellulose, demonstrating that the thermal stability of cellulose decreased after oxidation. The temperature at which the CS-DAC beads started to degrade was higher than that for the DAC beads. A similar increase in the temperature of initial degradation was not seen for the LC-DAC beads, most likely because of the difference in the amine group-containing compounds, since chitosan contains several amino groups in the molecule chain and can act as a crosslinking agent for DAC, while the single amino group in a molecule of $\mathrm{L}-$ cysteine cannot crosslink DAC molecules.

\section{Carbonization and characterization of CS-DAC and LC-DAC beads}

The purified, dried samples were pyrolyzed under nitrogen flow and then annealed to provide the nondoped, N-doped and N/S-co-doped carbon beads, named DAC-C (yield: 23.7\%), CS-DAC-C (yield: $21.5 \%$ ) and LC-DAC-C (yield: 18.5\%), respectively, as illustrated in Scheme 1. The morphology of the DAC-C, CS-DAC-C and LC-DAC-C beads was analyzed by SEM, as shown in Fig. 1d-f. Obviously, the surface morphology of DAC-C changed extensively after carbonization, to form a more compact surface with some small pores. Unexpectedly, the shape of most of the DAC beads was not maintained after carbonization and only a fraction of the DAC-C
DAC), and L-cysteine-functionalized DAC (LC-DAC) samples; c Raman spectra of carbonized DAC, CS-DAC, and LC-DAC samples

retained a spherical bead shape. Interestingly, the CSDAC-C and LC-DAC-C retained a spherical bead shape after pyrolyzation, which might have been the result of enhancement of the thermal stability as observed in the TGA curves (Fig. 2b), although this enhancement was small for LC-DAC. The porous surface morphology was maintained in the CS-DAC-C beads but disappeared from the LC-DAC-C beads (Fig. 1e, f), possibly because of the different thermal stabilities and the amine-containing reagent. Chitosan, a polyamine, served as a crosslinking reagent and can be considered as a spacer, as described by Lindh et al. (2016), to produce more porous beads whose structure was not totally broken during the carbonization. However, different processes can occur during the carbonization of LC-DAC, and these could have blocked the pores or caused the formation of smaller pores that could not be detected during the $\mathrm{N}_{2}$ sorption process, since the structure collapsed, as shown later.

Raman spectra of the carbonized cellulose beads are presented in Fig. 2c. All had very similar curves with two broad peaks located at $1335 \mathrm{~cm}^{-1}$ and $1590 \mathrm{~cm}^{-1}$. The D-band located at $1335 \mathrm{~cm}^{-1}$ corresponds to the defect-induced mode, while the G-band at $1590 \mathrm{~cm}^{-1}$ is associated with the vibration of $s p^{2}$ bonded carbon atoms in a $2 \mathrm{D}$ hexagonal lattice. The intensity ratio of the D-band to G-band are close. The similar spectra and the presence of a broad, lowintensity D-band indicate that the carbon in all the carbonized beads is composed of thin graphene layers with a low degree of graphitization, which may contribute to a good capacitive response, which in 
turn would be of interest for energy storage applications (Chen et al. 2014a; Wang et al. 2013b).

The element mapping images of CS-DAC-C and LC-DAC-C recorded by an EDX detector verified the existence of nitrogen and sulfur in the doped carbonized samples (Fig. 3a, b). The blue and purple spots in the element mapping images represent the presence of the nitrogen and sulfur elements, respectively, demonstrating N-doping and N/S-co-doping in CS-DAC-C and LC-DAC-C beads. Both blue and purple spots were distributed evenly throughout the entire samples, providing evidence that the nitrogen and sulfur elements were well dispersed in the carbonized bead samples.

XPS detection was used to investigate the surface chemical compositions and the nature of the elements in the composites further. As shown in Fig. 4a, C 1s $(285 \mathrm{eV})$ and $\mathrm{O} 1 \mathrm{~s}(531 \mathrm{eV})$ were clearly visible for all samples while $\mathrm{N} 1 \mathrm{~s}(398 \mathrm{eV})$ was observed in the spectra for CS-DAC, CS-DAC-C, LC-DAC and LCDAC-C. However, S $2 p(164 \mathrm{eV})$ was only seen for LC-DAC and LC-DAC-C. These results confirm the

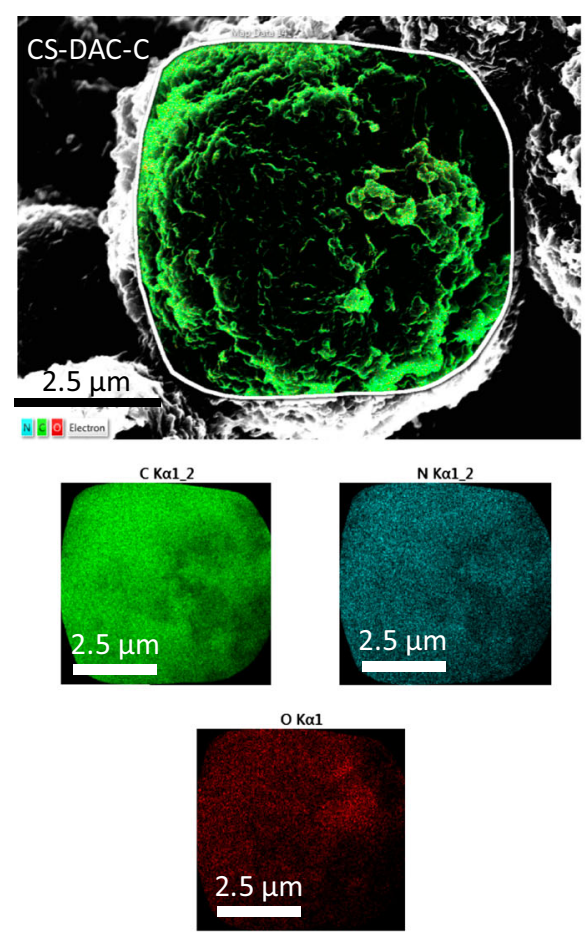

(a)

Fig. 3 Elemental analysis [C (green), O (red), N (blue) and S (purple)] of the composition of a carbonized chitosan-crosslinked 2,3-dialdehyde cellulose (CS-DAC-C) and b carbonized presence of nitrogen in the CS-DAC and CS-DAC-C samples, and of nitrogen and sulfur in the LC-DAC and LC-DAC-C samples. Figure $4 \mathrm{a}$ shows that the intensity of $\mathrm{C} 1 \mathrm{~s}$ in the carbonized samples became stronger at the expense of the intensity of $\mathrm{O} 1 \mathrm{~s}$ in the non-carbonized samples, indicating that the oxygen was consumed during pyrolysis. The high resolution spectra for $\mathrm{N} 1 \mathrm{~s}$ in Fig. 4b, c can be deconvoluted into two individual peaks at binding energies of 398.8 and $400.7 \mathrm{eV}$, assigned to pyridinic $\mathrm{N}$ and pyrrolic $\mathrm{N}$ (Artyushkova et al. 2013; Kelemen et al. 2002), respectively, which were produced during the carbonization process. The high resolution spectrum of $\mathrm{S}$ 2p (Fig. 4d) shows two peaks at 164.2 and $165.3 \mathrm{eV}$, assigned to $S 2 p_{3 / 2}$ and $S 2 p_{1 / 2}$, respectively, for the $\mathrm{C}-\mathrm{S}-\mathrm{C}-$ covalent bond, similar to thiophene-type sulfur (Qie et al. 2015).

To further understand the porous structure of the asprepared samples, $\mathrm{N}_{2}$ sorption isotherms were recorded and the pore volume distribution was evaluated for the carbonized beads, as shown in Fig. 5a, b. The SSA and pore-size distributions for all the
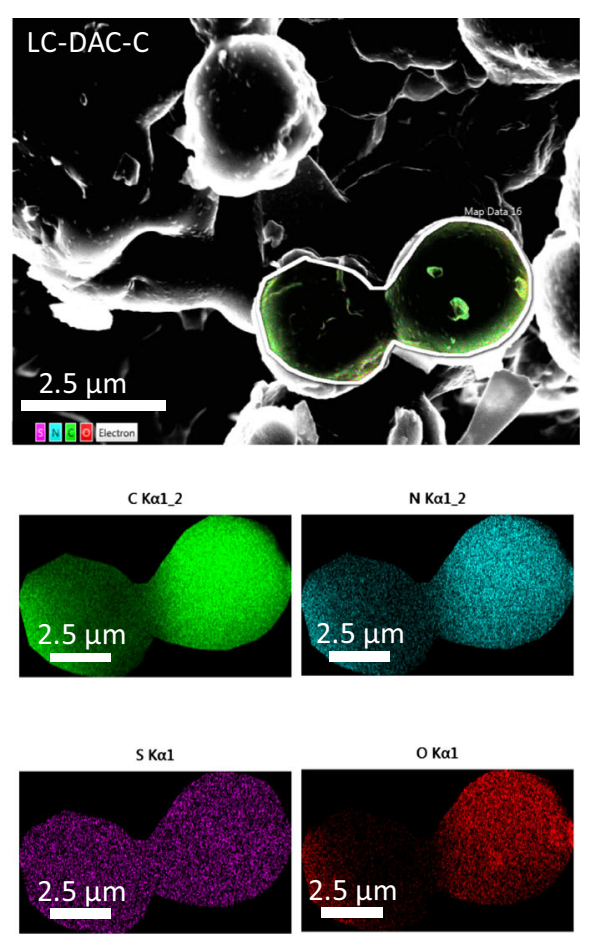

(b)

L-cysteine-functionalized 2,3-dialdehyde cellulose (LC-DACC) by energy dispersive X-ray spectroscopy. (Color figure online) 
Fig. 4 a X-ray

photoelectron spectroscopy survey spectra of the 2,3dialdehyde cellulose (DAC), carbonized DAC (DAC-C), chitosan-crosslinked DAC (CS-DAC), carbonized CSDAC (CS-DAC-C), L-cysteine-functionalized DAC (LC-DAC), and carbonized LC-DAC (LCDAC-C) samples, and fitted high-resolution spectra for b $\mathrm{N}$ 1s of CS-DAC-C, and c $\mathrm{N} 1 \mathrm{~s}$ and $\mathbf{d} \mathrm{S} 2 \mathrm{p}$ of LCDAC-C
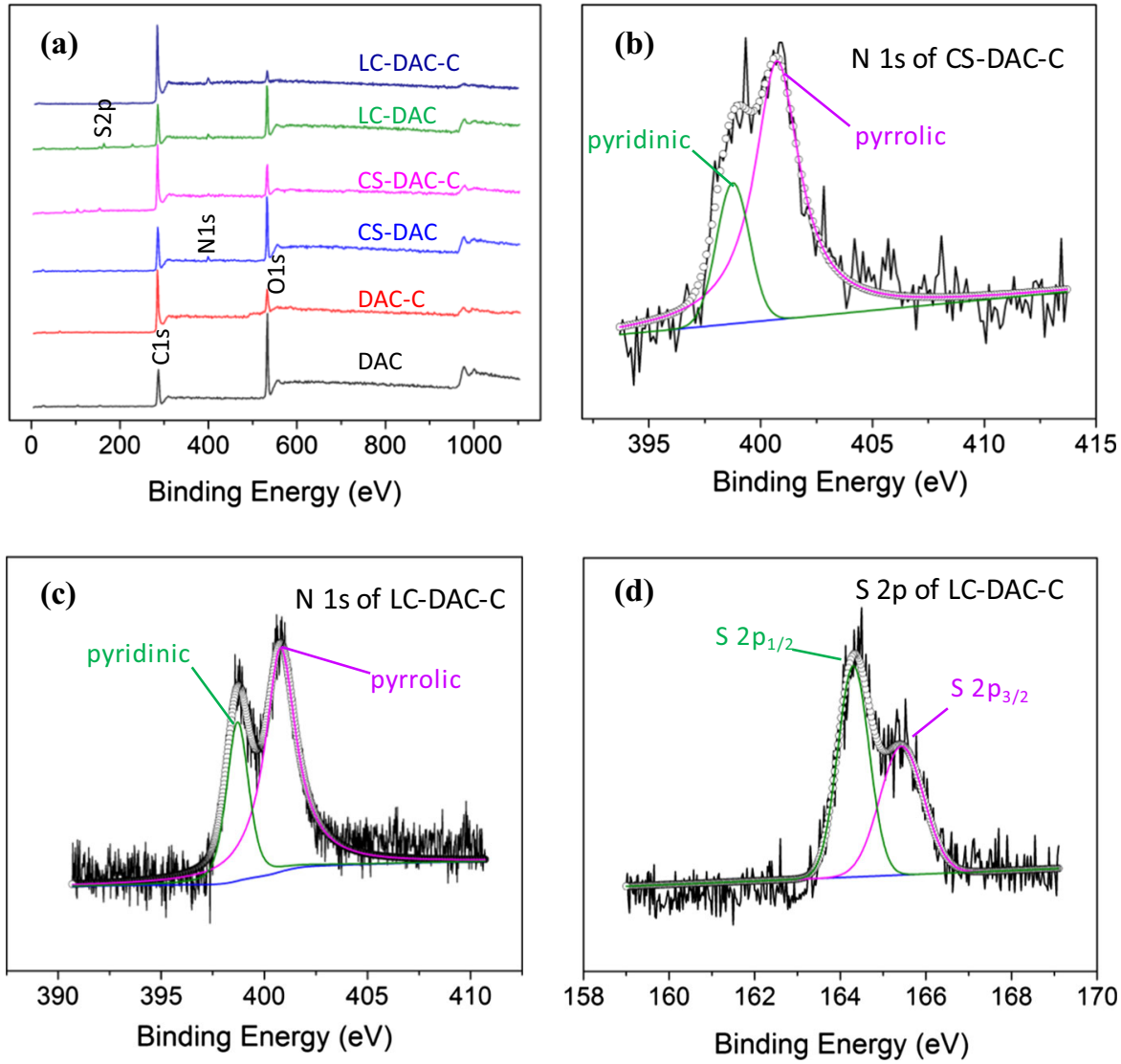

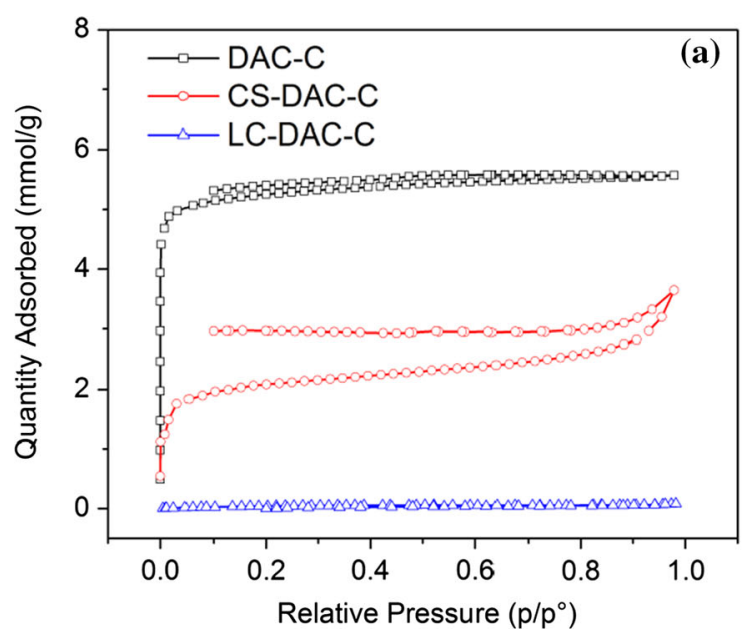

Fig. 5 a The nitrogen sorption isotherms $(T=77 \mathrm{~K})$ and b pore-size distribution graphs for pyrolyzed 2,3-dialdehyde cellulose (DAC-C), chitosan-crosslinked DAC (CS-DAC-C),

samples are summarized in Table 1. The steep increase at low pressure in the sorption isotherm for DAC-C (Fig. 5a) indicates that most of the pores in

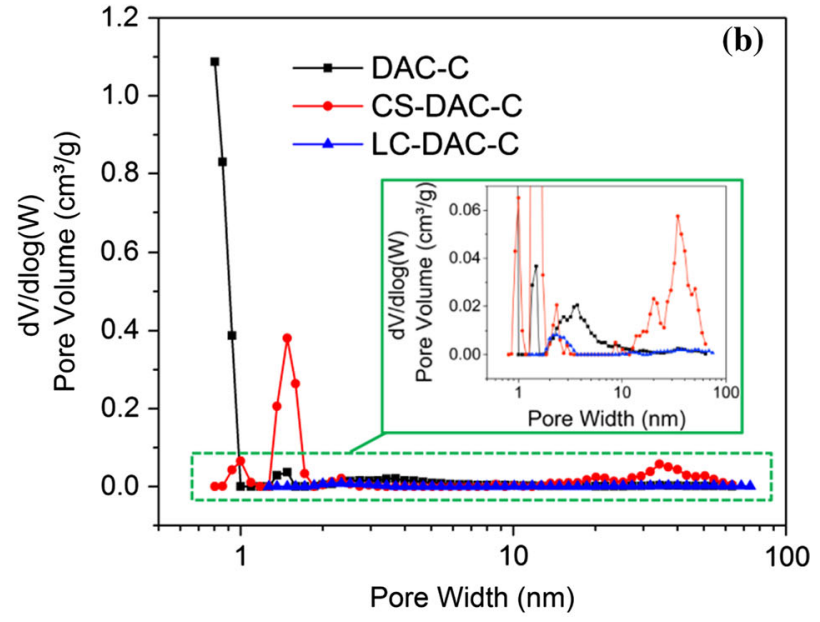

and L-cysteine-functionalized DAC (LC-DAC-C) beads. The inset in $\mathbf{b}$ presents an enlargement of the dashed part in the main panel

this material were micropores, while Fig. 5b suggests the presence of many small micropores with diameters below $1 \mathrm{~nm}$. In CS-DAC-C, the number of small 
Table 1 The specific surface areas (SSA) and pore volumes of 2,3-dialdehyde cellulose (DAC), carbonized DAC (DAC-C), chitosan-crosslinked DAC (CS-DAC), carbonized CS-DAC (CS-DAC-C), L-cysteine-functionalized DAC (LC-DAC), and carbonized LC-DAC (LC-DAC-C) bead samples

\begin{tabular}{lrl}
\hline Samples & SSA $\left(\mathrm{m}^{2} \mathrm{~g}^{-1}\right)$ & Pore volume $\left(\mathrm{cm}^{3} \mathrm{~g}^{-1}\right)$ \\
\hline DAC & $7.8 \pm 0.17$ & 0.013 \\
CS-DAC & $25.4 \pm 0.15$ & 0.094 \\
LC-DAC & $20.4 \pm 0.47$ & 0.039 \\
DAC-C & $414.5 \pm 6.28$ & 0.193 \\
CS-DAC-C & $167.8 \pm 1.93$ & 0.115 \\
LC-DAC-C & $4.2 \pm 0.07$ & 0.003 \\
\hline
\end{tabular}

micropores that were detectable by the instrument was significantly lower and some larger micropores (diameter around $1.5 \mathrm{~nm}$ ) and macropores appeared, which significantly reduced the SSA of CS-DAC-C (Table 1). The $\mathrm{N}_{2}$ sorption isotherm of LC-DAC-C (Fig. 5a) indicated nearly no nitrogen sorption and the pore volume of the micropores decreased by three orders of magnitude for LC-DAC-C compared to DAC-C. These results confirm the changes after carbonization observed with SEM (Fig. 1).

\section{Charge storage properties}

The capacitive performances of the carbon beads were firstly evaluated in a three-electrode configuration using $1 \mathrm{M} \mathrm{H}_{2} \mathrm{SO}_{4}$ as the electrolyte. Figure $6 \mathrm{a}$ presents the $\mathrm{CV}$ curves for the carbon beads at a scan rate of $10 \mathrm{mV} \mathrm{s}^{-1}$. All the carbon bead samples had similar quasi-rectangular shapes with small redox peaks; the pseudocapacitive behavior was in good agreement with the nearly linear galvanostatic charge and discharge (GCD) profiles (Fig. 6b). The CS-DAC$\mathrm{C}$ electrode had the largest $\mathrm{CV}$ loop area and the longest discharge time (compared to DAC-C and LCDAC-C). This electrode showed nearly linear GCD profiles even at high current densities up to $20 \mathrm{~A} \mathrm{~g}^{-1}$ (Fig. 6c), which indicates that the capacitive response (a)

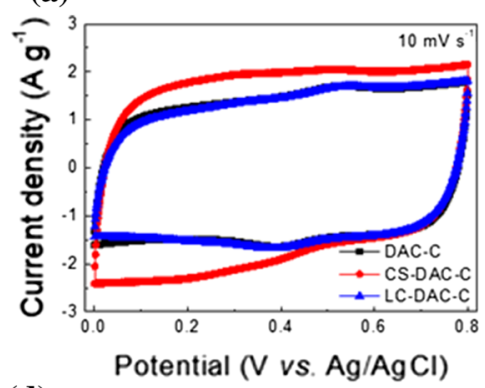

(d)

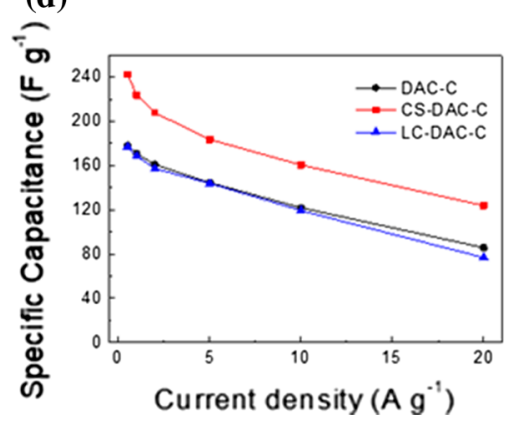

(b)

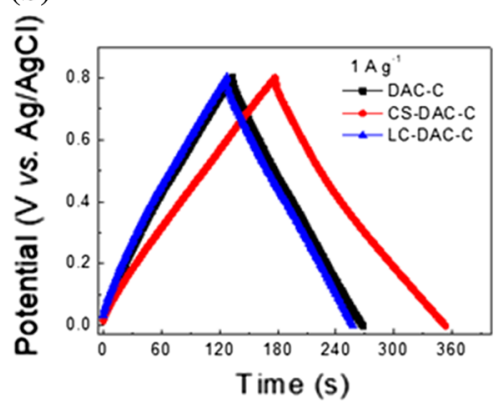

(e)

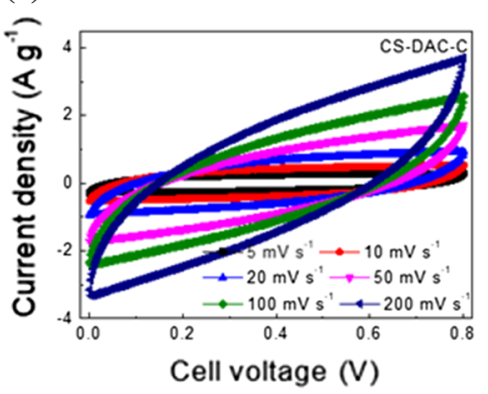

(c)

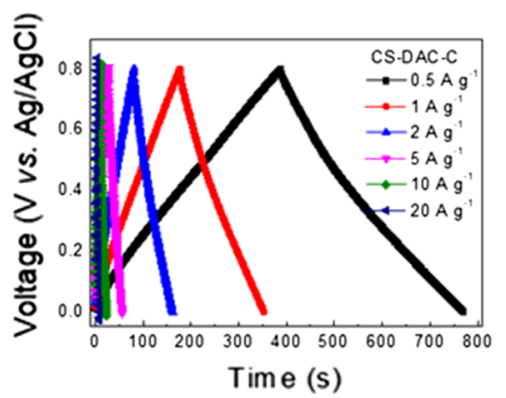

(f)

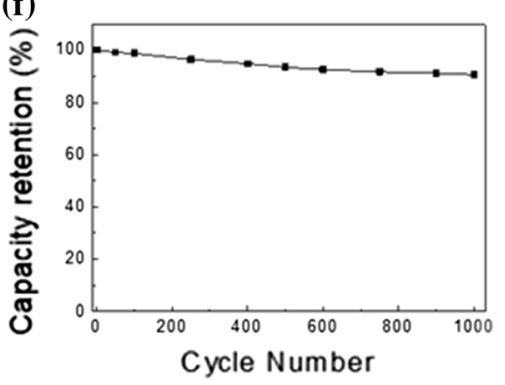

Fig. 6 a Voltammograms recorded at a scan rate of $10 \mathrm{mV} \mathrm{s}^{-1}$; b galvanostatic charge/discharge (GCD) curves obtained at a current density of $1 \mathrm{~A} \mathrm{~g} \mathrm{~g}^{-1}$ for the carbon beads in $1 \mathrm{M} \mathrm{H}_{2} \mathrm{SO}_{4}$ electrolyte; c GCD profiles for the CS-DAC-C electrode obtained at various current densities; $\mathbf{d}$ rate-dependent specific electrode capacitance for the carbon beads in a three-electrode setup; e cyclic voltammetry (CV) curves at different scan rates from 5 to $200 \mathrm{mV} \mathrm{s}^{-1}$ and $\mathbf{f}$ cycling performance recorded at a scan rate of $20 \mathrm{mV} \mathrm{s}^{-1}$ for the CS-DAC-C-based symmetric supercapacitor. $D A C$ 2,3-dialdehyde cellulose, $D A C$-C carbonized DAC, CS-DAC chitosan-crosslinked DAC, CS-DAC$C$ carbonized CS-DAC, $L C$-DAC L-cysteine-functionalized DAC, $L C-D A C-C$ carbonized LC-DAC 
was fast and the resistance was relatively low. As seen in Fig. 6d, the specific capacitance of CS-DAC-C (derived from the GCD curves) was up to $242 \mathrm{~F} \mathrm{~g}^{-1}$ at a current density of $1 \mathrm{~A} \mathrm{~g}^{-1}$, which was much higher than those obtained for DAC-C $\left(181 \mathrm{~F} \mathrm{~g}^{-1}\right)$ and LCDAC-C $\left(180 \mathrm{~F} \mathrm{~g} \mathrm{~g}^{-1}\right)$, and comparable to results previously reported for cellulose-based carbon electrodes (Chen et al. 2014a; Jiang et al. 2015, 2016; Li et al. 2016c). The higher capacitance of CS-DAC-C compared to LC-DAC-C was probably the result of the higher SSA of the former (Li et al. 2016a, b; Wang et al. 2017). The fact that the DAC-C electrode, which had the highest SSA of the samples under study, exhibited a lower capacity than CS-DAC-C indicates that the doped heteroatoms contributed more to the capacitive behavior than the SSA.

To evaluate the actual device performance of the CS-DAC-C electrode, further measurements were conducted in a symmetric supercapacitor with a twoelectrode configuration in $1 \mathrm{M} \mathrm{H}_{2} \mathrm{SO}_{4}$ electrolyte. The $\mathrm{CV}$ curve (Fig. 6e) maintained the relatively rectangular shape without apparent distortion even at a high scan rate of $200 \mathrm{mV} \mathrm{s}^{-1}$, indicating a good rate performance for the CS-DAC-C-based symmetric supercapacitor. The cell capacitance calculated from the $\mathrm{CV}$ curves of the symmetric supercapacitor was $42 \mathrm{~F} \mathrm{~g}^{-1}$ at a scan rate of $5 \mathrm{mV} \mathrm{s}^{-1}$. Moreover, the CS-DAC-C-based supercapacitor showed a remarkable cyclic durability, retaining over $91.5 \%$ of its initial capacitance even after 1000 cycles at a scan rate of $20 \mathrm{mV} \mathrm{s}^{-1}$ (Fig. 6b). The reasonable specific capacitance and good cycling performance of the CS-DAC-C beads indicates that cellulose beads offer a promising carbon source for supercapacitor applications.

\section{Conclusions}

Micrometer-sized, porous beads based on cellulose derivatives (CS-DAC and LC-DAC) were prepared by one-pot reductive amination of DAC beads followed by pyrolysis to obtain carbon beads doped with nitrogen (CS-DAC-C) and nitrogen and sulfur (LCDAC-C) and non-doped beads (DAC-C). Characterization by SEM, FTIR and TGA showed the changes in morphology, molecular groups and thermostability of the beads during/after the processes of modification and carbonization. XPS and EDX verified the nitrogen and sulfur doping, and the nitrogen sorption analysis results showed the different hierarchical porosities of the differently functionalized carbon beads. A suitable porosity and degree of nitrogen doping was achieved for CS-DAC-C beads and, when these were employed as the active material in electrodes, they showed the best specific capacitance of $242 \mathrm{~F} \mathrm{~g}^{-1}$ at a current density of $1 \mathrm{~A} \mathrm{~g}^{-1}$ and retained more than $91.5 \%$ of their initial capacitance after 1000 charge/ discharge cycles. The reasonable specific capacitance and good cycling performance of the CS-DAC-C beads indicate that the reductive amination of DAC beads is an effective approach to providing a heteroatom-doped carbon source whose corresponding carbonaceous active materials can be used as active electrode materials in energy storage.

Acknowledgments Chang-Qing Ruan is grateful for the financial support of the CSC (China Scholarship Council). We thank Zhen Qiu for her help during the measurement of Raman spectra.

Funding The Swedish Foundation for Strategic Research, the Olle Byggmästare Foundation, and the Bo Rydin Foundation.

\section{Compliance with ethical standards}

Conflict of interest The authors declare no competing financial interests.

Open Access This article is distributed under the terms of the Creative Commons Attribution 4.0 International License (http:// creativecommons.org/licenses/by/4.0/), which permits unrestricted use, distribution, and reproduction in any medium, provided you give appropriate credit to the original author(s) and the source, provide a link to the Creative Commons license, and indicate if changes were made.

\section{References}

Artyushkova K, Kiefer B, Halevi B, Knop-Gericke A, Schlogl R, Atanassov P (2013) Density functional theory calculations of XPS binding energy shift for nitrogen-containing graphene-like structures. Chem Commun 49:2539-2541. https://doi.org/10.1039/C3CC40324F

Biel B, Blase X, Triozon F, Roche S (2009) Anomalous doping effects on charge transport in graphene nanoribbons. Phys Rev Lett 102:096803. https://doi.org/10.1103/ PhysRevLett.102.096803

Brunauer S, Emmett PH, Teller E (1938) Adsorption of gases in multimolecular layers. J Am Chem Soc 60:309-319. https://doi.org/10.1021/ja01269a023

Chen LF, Huang ZH, Liang HW, Gao HL, Yu SH (2014a) Three-dimensional heteroatom-doped carbon nanofiber 
networks derived from bacterial cellulose for supercapacitors. Adv Funct Mater 24:5104-5111. https://doi.org/10. 1002/adfm.201400590

Chen X et al (2014b) Sulfur-doped porous reduced graphene oxide hollow nanosphere frameworks as metal-free electrocatalysts for oxygen reduction reaction and as supercapacitor electrode materials. Nanoscale 6:13740-13747. https://doi.org/10.1039/C4NR04783D

Deng Y, Xie Y, Zou K, Ji X (2016) Review on recent advances in nitrogen-doped carbons: preparations and applications in supercapacitors. J Mater Chem A 4:1144-1173. https://doi. org/10.1039/c5ta08620e

Duffy P et al (2012) Incandescent porous carbon microspheres to light up cells: solution phenomena and cellular uptake. J Mater Chem 22:432-439. https://doi.org/10.1039/ C1JM14303D

Fan H, Shen W (2015) Carbon nanosheets: synthesis and application. Chemsuschem 8:2004-2027. https://doi.org/ $10.1002 /$ cssc. 201500141

Ghosh A, Lee YH (2012) Carbon-based electrochemical capacitors. Chemsuschem 5:480-499. https://doi.org/10. 1002/cssc.201100645

Jeong GH, Baek S, Lee S, Kim S-W (2016) Metal oxide/graphene composites for supercapacitive electrode materials. Chem Asian J 11:949-964. https://doi.org/10.1002/ asia. 201501072

Jiang H, Lee PS, Li C (2013) 3D carbon based nanostructures for advanced supercapacitors. Energy Environ Sci 6:41-53. https://doi.org/10.1039/C2EE23284G

Jiang L, Nelson GW, Kim H, Sim I, Han SO, Foord JS (2015) Cellulose-derived supercapacitors from the carbonisation of filter paper. ChemistryOpen 4:586-589. https://doi.org/ 10.1002/open.201500150

Jiang L, Nelson GW, Han SO, Kim H, Sim IN, Foord JS (2016) Natural cellulose materials for supercapacitors. Electrochim Acta 192:251-258. https://doi.org/10.1016/j. electacta.2015.12.138

Kelemen SR, Afeworki M, Gorbaty ML, Kwiatek PJ, Solum MS, Hu JZ, Pugmire RJ (2002) XPS and 15 N NMR study of nitrogen forms in carbonaceous solids. Energy Fuels 16:1507-1515. https://doi.org/10.1021/ef0200828

Klemm D, Heublein B, Fink HP, Bohn A (2005) Cellulose: fascinating biopolymer and sustainable raw material. Angew Chem Int Ed 44:3358-3393. https://doi.org/10. 1002/anie.200460587

Lee JW, Ko JM, Kim J-D (2012) Hydrothermal preparation of nitrogen-doped graphene sheets via hexamethylenetetramine for application as supercapacitor electrodes. Electrochim Acta 85:459-466. https://doi.org/10.1016/j. electacta.2012.08.070

Li W et al (2007) Nitrogen enriched mesoporous carbon spheres obtained by a facile method and its application for electrochemical capacitor. Electrochem Commun 9:569-573. https://doi.org/10.1016/j.elecom.2006.10.027

Li M, Liu C, Cao H, Zhao H, Zhang Y, Fan Z (2014) KOH selftemplating synthesis of three-dimensional hierarchical porous carbon materials for high performance supercapacitors. J Mater Chem A 2:14844-14851. https://doi.org/ 10.1039/C4TA02167C

Li B, Dai F, Xiao Q, Yang L, Shen J, Zhang C, Cai M (2016a) Activated carbon from biomass transfer for high-energy density lithium-ion supercapacitors. Adv Energy Mater. https://doi.org/10.1002/aenm.201600802

Li B, Dai F, Xiao Q, Yang L, Shen J, Zhang C, Cai M (2016b) Nitrogen-doped activated carbon for a high energy hybrid supercapacitor. Energy Environ Sci 9:102-106. https://doi. org/10.1039/c5ee03149d

Li Z, Liu J, Jiang K, Thundat T (2016c) Carbonized nanocellulose sustainably boosts the performance of activated carbon in ionic liquid supercapacitors. Nano Energy 25:161-169. https://doi.org/10.1016/j.nanoen.2016.04.036

Li M, Zong L, Li X, You J, Wu X, Kong Q, Li C (2017a) Fibrous carbon nanosheets from Kevlar nanofibrils: compromising one and two dimensions of carbon nanomaterials for optimal capacitive performance. Carbon 123:565-573. https://doi.org/10.1016/j.carbon.2017.08.009

Li Z et al (2017b) Carbon materials derived from chitosan/cellulose cryogel-supported zeolite imidazole frameworks for potential supercapacitor application. Carbohydr Polym 175:223-230. https://doi.org/10.1016/j.carbpol.2017.07. 089

Lindh J, Carlsson DO, Strømme M, Mihranyan A (2014) Convenient one-pot formation of 2,3-dialdehyde cellulose beads via periodate oxidation of cellulose in water. Biomacromol 15:1928-1932. https://doi.org/10.1021/ bm5002944

Lindh J, Ruan C, Strømme M, Mihranyan A (2016) Preparation of porous cellulose beads via introduction of diamine spacers. Langmuir 32:5600-5607. https://doi.org/10.1021/ acs.langmuir.6b01288

Liu M et al (2013) Nickel-doped activated mesoporous carbon microspheres with partially graphitic structure for supercapacitors. Energy Fuels 27:1168-1173. https://doi.org/10. 1021/ef302028j

Ma X et al (2014) Mesoporous size controllable carbon microspheres and their electrochemical performances for supercapacitor electrodes. J Mater Chem A 2:8407-8415. https://doi.org/10.1039/C4TA00333K

Qie L, Chen W, Xiong X, Hu C, Zou F, Hu P, Huang Y (2015) Sulfur-doped carbon with enlarged interlayer distance as a high-performance anode material for sodium-ion batteries. Adv Sci 2:1500195. https://doi.org/10.1002/advs. 201500195

Rinaudo M (2006) Chitin and chitosan: properties and applications. Prog Polym Sci 31:603-632. https://doi.org/10. 1016/j.progpolymsci.2006.06.001

Ruan CQ, Strømme M, Lindh J (2016) A green and simple method for preparation of an efficient palladium adsorbent based on cysteine functionalized 2,3-dialdehyde cellulose. Cellulose 23:2627-2638. https://doi.org/10.1007/s10570016-0976-0

Ruan C-Q, Strømme M, Lindh J (2018) Preparation of porous 2,3-dialdehyde cellulose beads crosslinked with chitosan and their application in adsorption of Congo red dye. Carbohydr Polym 181:200-207. https://doi.org/10.1016/j. carbpol.2017.10.072

Simon P, Gogotsi Y, Dunn B (2014) Where do batteries end and supercapacitors begin? Science 343:1210-1211. https:// doi.org/10.1126/science. 1249625

Wang G, Zhang L, Zhang J (2012) A review of electrode materials for electrochemical supercapacitors. Chem Soc Rev 41:797-828. https://doi.org/10.1039/c1cs15060j 
Wang L, Ji H, Wang S, Kong L, Jiang X, Yang G (2013a) Preparation of $\mathrm{Fe} 3 \mathrm{O} 4$ with high specific surface area and improved capacitance as a supercapacitor. Nanoscale 5:3793-3799. https://doi.org/10.1039/C3NR00256J

Wang Z, Xiong X, Qie L, Huang Y (2013b) High-performance lithium storage in nitrogen-enriched carbon nanofiber webs derived from polypyrrole. Electrochim Acta 106:320-326. https://doi.org/10.1016/j.electacta.2013.05.088

Wang Z, Tammela P, Zhang P, Strømme M, Nyholm L (2014) High areal and volumetric capacity sustainable all-polymer paper-based supercapacitors. J Mater Chem A 2:16761-16769. https://doi.org/10.1039/C4TA03724C

Wang ZH, Carlsson DO, Tammela P, Hua K, Zhang P, Nyholm L, Strømme M (2015) Surface modified nanocellulose fibers yield conducting polymer-based flexible supercapacitors with enhanced capacitances. ACS Nano 9:7563-7571. https://doi.org/10.1021/acsnano.5b02846

Wang Z, Tammela P, Strømme M, Nyholm L (2017) Cellulosebased supercapacitors: material and performance considerations. Adv Energy Mater 7:1700130. https://doi.org/10. 1002/aenm.201700130

Winter M, Brodd RJ (2004) What are batteries, fuel cells, and supercapacitors? Chem Rev 104:4245-4270. https://doi. org/10.1021/cr020730k

Wohlgemuth S-A, Vilela F, Titirici M-M, Antonietti M (2012) A one-pot hydrothermal synthesis of tunable dual heteroatom-doped carbon microspheres. Green Chem 14:741-749. https://doi.org/10.1039/C2GC16415A

$\mathrm{Xu}$ GY et al (2015) Biomass-derived porous carbon materials with sulfur and nitrogen dual-doping for energy storage. Green Chem 17:1668-1674. https://doi.org/10.1039/ c4gc02185a

Xu MM, Huang QB, Sun RC, Wang XH (2016) Simultaneously obtaining fluorescent carbon dots and porous active carbon for supercapacitors from biomass. RSC Adv 6:88674-88682. https://doi.org/10.1039/c6ra18725k

You B, Wang L, Yao L, Yang J (2013) Three dimensional $\mathrm{N}$-doped graphene-CNT networks for supercapacitor. Chem Commun 49:5016-5018. https://doi.org/10.1039/ C3CC41949E

You J, Li M, Ding B, Wu X, Li C (2017) Crab chitin-based 2D soft nanomaterials for fully biobased electric devices. Adv Mater 29:1606895. https://doi.org/10.1002/adma. 201606895

Zhai Y, Dou Y, Zhao D, Fulvio PF, Mayes RT, Dai S (2011) Carbon materials for chemical capacitive energy storage. Adv Mater 23:4828-4850. https://doi.org/10.1002/adma. 201100984

Zhao Q et al (2014a) Supercapacitive performance of hierarchical porous carbon microspheres prepared by simple onepot method. J Power Sources 254:10-17. https://doi.org/ 10.1016/j.jpowsour.2013.12.091

Zhao Y, Liu M, Gan L, Ma X, Zhu D, Xu Z, Chen L (2014b) Ultramicroporous carbon nanoparticles for the high-performance electrical double-layer capacitor electrode. Energy Fuels 28:1561-1568. https://doi.org/10.1021/ ef402070j

Zhao D et al (2017) Highly flexible and conductive cellulosemediated PEDOT:PSS/MWCNT composite films for supercapacitor electrodes. ACS Appl Mater Interfaces 9:13213-13222. https://doi.org/10.1021/acsami.7b01852

Zhu D et al (2015) Nitrogen-containing carbon microspheres for supercapacitor electrodes. Electrochim Acta 158:166-174. https://doi.org/10.1016/j.electacta.2015.01.155

Zong L, Wu X, You J, Li M, Li C (2016) Modulating structural hierarchies of manganese oxide in morphology and porosity by marine biopolymer for improved supercapacitors. Electrochim Acta 213:709-716. https://doi.org/10. 1016/j.electacta.2016.07.158 